\title{
Effect of Electric Field on Ferroelectric and Dielectric Properties in Rochelle Salt Crystal
}

\author{
KALIKA PRASAD SEMWAL and TRILOK CHANDRA UPADHYAY
}

Physics Department, H.N.B. Garhwal University (A Central University) Srinagar (Garhwal), Uttarakhand- 246174, India

kalikaprasadsemwal@gmail.com

Received 20 December 2016 / Accepted 15 January 2017

\begin{abstract}
The model of two-sublattice pseudospin lattice coupled mode for Rochelle salt has been modified by adding third and fourth-order phonon anharmonic interaction terms and electric field term. By using double-time temperature dependent Green's function method, expressions for shift, width, soft mode frequency, dielectric constant and loss tangent were obtained for Rochelle salt crystal. By fitting model values of physical quantities, temperature dependence of soft mode frequency, dielectric constant and loss tangent have been calculated for different fields for Rochelle salt. Theoretical results agree with the experimental results of others.
\end{abstract}

Keywords: Ferroelectrics, Green's function, Soft mode, Anharmonic interaction

\section{Introduction}

Potassium sodium tartrate $\left(\mathrm{NaKC}_{4} \mathrm{H}_{4} \mathrm{O}_{6} 4 \mathrm{H}_{2} \mathrm{O}\right)$ or Rochelle salt (RS) is the material in which ferroelectricity was discovered earliest. Large crystals of Rochelle salt are easy to grow. Although, it is the earliest ferroelectric material but is still the subject of intensive study due to its two transitions. It is ferroelectric between $255 \mathrm{~K}$ to $297 \mathrm{~K}$ showing monoclinic structure in ferroelectric phase. On duteration, the transition temperatures shift to $251 \mathrm{~K}$ and $306 \mathrm{~K}$ respectively. The Theories of ferroelectric properties of Rochelle salt were initiated by Muller ${ }^{1}$. Mason ${ }^{2}$ assumed that the displacement of the proton is the $\mathrm{O}-\left(\mathrm{H}_{2} \mathrm{O}\right)_{10}$ hydrogen bond is the ferroelectric dipole and was able to obtain two curie points in agreement with observation. Chaudhuri et al., ${ }^{3}$ have used two-sublattice-pseudospin-lattice coupled mode model along with a fourth-order phonon anharmonic term. However, they decoupled the correlations at an early stage and neglected third-order anharmonic interaction term. They, therefore, could not obtain better and convincing results. Hlinka et al., ${ }^{4}$ Shiozaki et al., ${ }^{5}$ Noda et al., ${ }^{6}$ Kikuta et al., ${ }^{7}$ have experimentally studied dielectric and other properties of Rochelle salt crystal. In the present work an external electric field term, third-order phonon anharmonic interaction term and fourth-order phonon anharmonic interaction term, have been added in the two-sublattice pseudospin-lattice coupled mode model. By applying double time thermal Green's function $\operatorname{method}^{8}$, expressions for shift, width, renormalized 
soft mode frequency, dielectric constant and loss tangent have been evaluated. By using model values of various physical quantities given by Chaudhuri et al. ${ }^{3}$ temperature and field dependences of soft mode frequency, dielectric constant and loss tangent have been calculated. The theoretical results have been compared with experimental data of Sandy and Jones $^{9}$ for Rochelle salt.

\section{Model Hamiltonian}

For Rochelle salt, the extended two-sublattice pseudospin-lattice coupled mode model, along with third-and fourth-order phonon anharmonic interaction terms is expressed as

$$
\begin{aligned}
& H=-2 \Omega \sum_{i}\left(S_{1 i}^{x}+S_{2 i}^{x}\right)-\sum_{i j} J_{i j}\left[\left(S_{1 i}^{z} S_{1 j}^{z}\right)\right. \\
& \left.+\left(S_{2 i}^{z} S_{2 j}^{z}\right)\right]-\sum_{i j} K_{i j}\left(S_{1 i}^{z} S_{2 j}^{z}\right)-\Delta \sum_{i}\left(S_{1 i}^{z}+S_{2 i}^{z}\right)-2 \mu E \sum_{i}\left(S_{1 i}^{z}+S_{2 i}^{z}\right) \\
& -\sum_{i k} V_{i k} S_{1 i}^{z} A_{k}-\sum_{i k} V_{i k} S_{2 i}^{z} A_{k}^{+}
\end{aligned}
$$

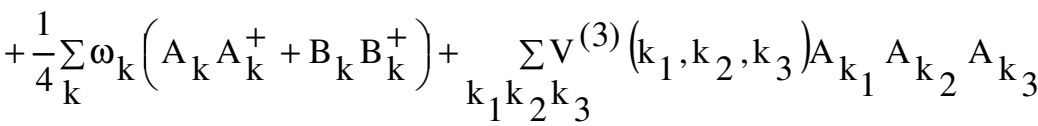

$$
\begin{aligned}
& +\mathrm{k}_{1} \mathrm{k}_{2} \mathrm{k}_{3} \mathrm{k}_{4}^{(4)}\left(\mathrm{k}_{1}, \mathrm{k}_{2}, \mathrm{k}_{3} \mathrm{k}_{4}\right) \mathrm{A}_{\mathrm{k}_{1}} \mathrm{~A}_{\mathrm{k}_{2}} \mathrm{~A}_{\mathrm{k}_{3}} \mathrm{~A}_{\mathrm{k}_{4}}
\end{aligned}
$$

Where $\Omega$ is proton tunnelling frequency, $S^{z}$ and $S^{x}$ are components of pseudospin variables, $J_{\mathrm{ij}}$ is exchange interaction constant between spin of same lattices, $\mathrm{K}_{\mathrm{ij}}$ is exchange interaction constant between spins of neighbouring lattices, $V_{i k}$ is spin-lattice interaction constant, $\mu$ is dipole moment of $\mathrm{O}-\mathrm{H} \ldots \mathrm{O}$ bond, $\mathrm{A}_{\mathrm{k}}$ and $\mathrm{B}_{\mathrm{k}}$ are position and momentum operators, $\omega_{\mathrm{k}}$ is harmonic phonon frequency, $\mathrm{V}^{(3)}$ and $\mathrm{V}^{(4)}$ are third-and fourth-order atomic force constants ${ }^{10,11}$.

\section{Green's function}

We consider the Green's function (GF)

$$
\mathrm{G}_{\mathrm{ij}}\left(\mathrm{t}-\mathrm{t}^{\prime}\right)=\left\langle\left\langle\mathrm{S}_{1 \mathrm{i}}^{\mathrm{Z}}(\mathrm{t}) ; \mathrm{S}_{1 \mathrm{j}}^{\mathrm{Z}}\left(\mathrm{t}^{\prime}\right)\right\rangle\right\rangle=-i \theta\left(t-t^{\prime}\right)\left\langle\left[S_{1 i}^{z}(t) ; S_{1 j}^{z}\left(t^{\prime}\right)\right]\right\rangle
$$

Differentiating Eq. (2) with respect to time $t$ and t' two times each using Hamiltonian (1), Fourier transforming it and writing into Dyson's equation form we obtain

$$
G_{i j}(\omega)=\frac{\Omega\left\langle S_{1 i}{ }^{x}\right\rangle}{\pi\left[\omega^{2}-\widetilde{\Omega}^{2}-P(\omega)\right]}
$$

Where $\widetilde{\Omega}^{2}=a^{\prime 2}+b^{2}+b c$

$$
\begin{aligned}
a^{\prime} & =\left[J\left\langle S_{1}^{x}\right\rangle+K\left\langle S_{2}^{z}\right\rangle+2 \mu E+\Delta\right] \\
\mathrm{b} & =2 \Omega \\
\text { and } \mathrm{c}= & =\left[j\left\langle s_{1}{ }^{x}\right\rangle+k\left\langle s_{2}{ }^{x}\right\rangle\right]
\end{aligned}
$$


Now $P(\omega)$ contains higher order Green's functions

$$
\langle a b c d\rangle=\langle a b\rangle\langle c d\rangle+\langle a c\rangle\langle b d\rangle+\langle a d\rangle\langle b c\rangle
$$

The simpler Green's functions are solved in zeroth-order approximation, i.e. higher order Green's functions are neglected.

Shift, Width and Ferroelectric mode frequency

Then $P(\omega)$ is resolved into its real $\Delta(\omega)$ and imaginary parts $\Gamma(\omega)$. The Green's function given in Eq. 3 finally becomes

$$
\begin{aligned}
& G_{i j}(\omega)=\frac{\Omega\left\langle S_{1 i}{ }^{x}\right\rangle \delta_{i j}}{\pi\left[\omega^{2}-\Omega^{2}-2 \Omega\{\Delta(\omega)+i \Gamma(\omega)\}\right]} \\
& \text { with } \Delta(\omega)=\Delta_{1}(\omega)+\Delta_{2}(\omega)+\Delta_{3}(\omega)+\Delta_{4}(\omega) \\
& \Delta_{1}(\omega)=\frac{a^{\prime 4}}{2 \Omega\left(\omega^{2}-\widetilde{\Omega}^{2}\right)} \\
& \Delta_{2}(\omega)=\frac{V_{i k}^{2} N_{k} a^{2}}{2 \Omega\left(\omega^{2}-\widetilde{\Omega}^{2}\right)} \\
& \Delta_{3}(\omega)=\frac{2 V_{i k}^{2}\left\langle S_{1}^{x}\right\rangle \omega_{k}\left(\omega^{2}-\tilde{\widetilde{\omega}}_{k}^{2}\right)}{\left[\left(\omega^{2}-\tilde{\widetilde{\omega}}_{k}^{2}\right)^{2}+4 \omega_{k}^{2} \Gamma_{k}^{2}(\omega)\right]} \\
& \Delta_{4}(\omega)=\frac{4 \mu^{2} E^{2} a^{\prime 2}}{2 \Omega\left(\omega^{2}-\widetilde{\Omega}^{2}\right)} \\
& \text { and } \Gamma(\omega)=\Gamma_{1}(\omega)+\Gamma_{2}(\omega)+\Gamma_{3}(\omega)+\Gamma_{4}(\omega) \\
& \Gamma_{1}(\omega)=\frac{\pi a^{\prime 4}}{4 \Omega \tilde{\Omega}}[\delta(\omega-\tilde{\Omega})-\delta(\omega+\tilde{\Omega})] \\
& \Gamma_{2}(\omega)=\frac{V_{i k}^{2} N_{k} a^{\prime 2}}{4 \Omega \tilde{\Omega}}[\delta(\omega-\tilde{\Omega})-\delta(\omega+\tilde{\Omega})] \\
& \Gamma_{3}(\omega)=\frac{4 V_{i k}^{2}\left\langle S_{1}^{x}\right\rangle\left(\omega^{2}-\tilde{\widetilde{\omega}}_{k}^{2}\right)}{\left[\left(\omega^{2}-\tilde{\tilde{\omega}}_{k}^{2}\right)^{2}+4 \omega_{k}^{2} \Gamma_{k}^{2}(\omega)\right]} \\
& \Gamma_{4}(\omega)=\frac{4 \pi \mu^{2} E^{2} a^{\prime 2}}{4 \Omega \tilde{\Omega}}[\delta(\omega-\tilde{\Omega})-\delta(\omega+\widetilde{\Omega})]
\end{aligned}
$$


In Eqs 13 and 18, $\tilde{\widetilde{\omega}}_{k}$ is renormalized phonon frequency and $\Gamma_{k}(\omega)$ is phonon width. These are obtained by solving phonon Green's function

$$
\begin{aligned}
& G_{k k^{\prime}}\left(t-t^{\prime}\right)=\left\langle\left\langle A_{k}(t) ; A_{k}^{+}\left(t^{\prime}\right)\right\rangle\right\rangle \text {. } \\
& \Delta_{k}(\omega)=\operatorname{Re} P(\omega) \\
& =18 P \sum_{k_{1} k_{2}}\left|V^{(3)}\left(k_{1}, k_{2}-k\right)\right|^{2} \\
& \frac{\omega_{\mathrm{k}_{1}} \omega_{\mathrm{k}_{2}}}{\widetilde{\omega}_{\mathrm{k}_{1}} \widetilde{\omega}_{\mathrm{k}_{2}}}\left\{\left(\mathrm{n}_{\mathrm{k}_{1}}+\mathrm{n}_{\mathrm{k} 2}\right) \frac{\widetilde{\omega}_{\mathrm{k}_{1}}+\widetilde{\omega}_{\mathrm{k}_{2}}}{\omega^{2}-\left(\widetilde{\omega}_{\mathrm{k}_{1}}+\widetilde{\omega}_{\mathrm{k}_{2}}\right)^{2}}\right. \\
& \left.+\left(n_{k_{2}}+n_{k_{1}}\right) \frac{\widetilde{\omega}_{k_{1}}+\widetilde{\omega}_{k_{2}}}{\omega^{2}-\left(\widetilde{\omega}_{k_{1}}+\widetilde{\omega}_{k_{2}}\right)^{2}}\right\} \\
& +48 \mathrm{P} \sum_{\mathrm{k} 1_{1} \mathrm{k}_{2 \mathrm{k}_{3}}}\left|\mathrm{~V}^{(4)}\left(\mathrm{k}_{1}, \mathrm{k}_{2}, \mathrm{k}_{3},-\mathrm{k}\right)\right|^{2} \frac{\omega_{\mathrm{k}_{1}} \omega_{\mathrm{k}_{2}} \omega_{\mathrm{k}_{3}}}{\widetilde{\omega}_{\mathrm{k}_{1}} \tilde{\omega}_{\mathrm{k}_{2}} \widetilde{\omega}_{\mathrm{k}_{3}}} \\
& \left\{\left(1+\mathrm{n}_{\mathrm{k}_{1}} \mathrm{n}_{\mathrm{k}_{2}}+\mathrm{n}_{\mathrm{k}_{2}} \mathrm{n}_{\mathrm{k}_{3}}+\mathrm{n}_{\mathrm{k}_{3}} \mathrm{n}_{\mathrm{k}_{1}}\right) \frac{\tilde{\omega}_{\mathrm{k}_{1}}+\widetilde{\omega}_{\mathrm{k}_{2}}+\tilde{\omega}_{\mathrm{k}_{3}}}{\omega^{2}-\left(\tilde{\omega}_{\mathrm{k}_{1}}+\tilde{\omega}_{\mathrm{k}_{2}}+\tilde{\omega}_{\mathrm{k}_{3}}\right)^{2}}\right. \\
& +3\left(1-\mathrm{n}_{\mathrm{k}_{2}} \mathrm{n}_{\mathrm{k}_{1}}+\mathrm{n}_{\mathrm{k} 2} \mathrm{n}_{\mathrm{k}_{3}}-\mathrm{n}_{\mathrm{k}_{3}} \mathrm{n}_{\mathrm{k}_{1}}\right) \\
& \frac{\widetilde{\omega}_{\mathrm{k}_{1}}+\widetilde{\omega}_{\mathrm{k} 2}+\widetilde{\omega}_{\mathrm{k} 3}}{\omega^{2}-\left(\widetilde{\omega}_{\mathrm{k}_{1}}+\widetilde{\omega}_{\mathrm{k}_{2}}+\widetilde{\omega}_{\mathrm{k} 3}\right)^{2}}+(\text { higher terms }) \\
& \text { and } \\
& \Gamma_{k}(\omega)=9 \pi \sum_{k_{1} k_{2}}\left|V^{(3)}\left(k_{1}, k_{2},-k\right)\right|^{2} \frac{\omega_{k_{1}} \omega_{k_{2}}}{\widetilde{\omega}_{k_{1}} \widetilde{\omega}_{k_{2}}} \\
& \left\{( n _ { k _ { 1 } } + n _ { k _ { 2 } } ) \left[\delta\left(\omega+\widetilde{\omega}_{k_{1}}+\widetilde{\omega}_{k_{1}}\right)\right.\right. \\
& -\left(\omega-\widetilde{\omega}_{k_{1}}-\widetilde{\omega}_{k_{1}}\right) \\
& \left.+\left(n_{k_{2}}-n_{k_{1}}\right)\left[\delta\left(\omega+\widetilde{\omega}_{k_{1}}+\widetilde{\omega}_{k_{2}}\right)-\delta\left(\omega+\widetilde{\omega}_{k_{1}}+\widetilde{\omega}_{k_{2}}\right)\right]\right\} \\
& +48 \pi \sum_{k_{1} k_{2} k_{3}}\left|V^{(4)}\left(k_{1}, k_{2}, k_{3}, k_{4}\right)\right|^{2} \\
& X\left\{\left(1+n_{k_{1}} n_{k_{2}}+n_{k_{2}} n_{k_{3}}+n_{k_{3}} n_{k_{4}}\right)\right. \\
& X\left[\delta\left(\omega+\widetilde{\omega}_{k_{1}}+\widetilde{\omega}_{k_{2}}+\widetilde{\omega}_{k_{3}}\right)-\left(\omega-\widetilde{\omega}_{k_{1}}-\widetilde{\omega}_{k_{2}}-\widetilde{\omega}_{k_{3}}\right)\right] \\
& +3\left(n_{k_{1}} n_{k_{2}}+n_{k_{2}} n_{k_{3}}-n_{k_{3}} n_{k_{4}}\right)
\end{aligned}
$$




$$
\widetilde{\widetilde{\omega}}_{k}{ }^{2}=\widetilde{\omega}_{k}{ }^{2}+A_{k}(T)
$$

Now Green's function (9) becomes

$$
\begin{aligned}
& G_{i j}(\omega)=\frac{\Omega\left\langle S_{1 i}^{x}\right\rangle \delta_{i j}}{\pi\left[\omega^{2}-\tilde{\Omega}^{2}-2 \Omega i \Gamma(\omega)\right]} \\
& \text { With } \quad \tilde{\Omega}^{2}=\widetilde{\Omega}^{2}+2 \Omega \Delta(\omega)
\end{aligned}
$$

Solving Eq 24 self consistently one gets

$$
\tilde{\Omega}_{-}{ }^{2}=\frac{1}{2}\left[\left(\tilde{\widetilde{\omega}}_{k}{ }^{2}+\tilde{\widetilde{\Omega}}^{2}\right) \pm\left(\tilde{\widetilde{\omega}}_{k}^{2}-\widetilde{\widetilde{\Omega}}^{2}\right)^{2}+8 V_{i k}\left\langle S_{1 i}{ }^{x}\right\rangle \Omega \omega_{k}\right]^{\frac{1}{2}}
$$

\section{Dielectric constant and loss tangent}

The response of a crystal to electromagnetic field is expressed by electrical susceptibility given by

$$
\chi=-\lim _{\in \rightarrow 0} 2 \pi N \mu^{2} \mathrm{G}_{\mathrm{ij}}(\omega+\mathrm{i} \in)
$$

Where $\mathrm{N}$ is number of dipoles having dipole moment $\mu$ in the sample. The dielectric constant $\in(\omega)$ is related to susceptibility as

$$
\in=1+4 \pi \chi
$$

We have from Eqs 26, 27 and 23

since $[\in(\omega)\rangle\rangle 1]$, in ferroelectrics .

$$
\in(\omega)=\frac{\left(-8 \pi N \mu^{2}\right)\left\langle S_{1 j}^{x}\right\rangle \Omega \delta_{i j}}{\left[\omega^{2}-\widetilde{\Omega}^{2}-2 \Omega i \Gamma(\omega)\right]}
$$

Eq. 28 shows that dielectric constant explicitly depends upon electric field through $\widetilde{\Omega}$.The dissipation of power in dielectrics is expressed as tangent losss given by

$$
\tan \delta=\frac{\operatorname{Im} \text { aginary } \epsilon}{\operatorname{Re} a l \in}=-\frac{2 \Omega \Gamma(\omega)}{\left(\omega^{2}-\widetilde{\Omega}^{2}\right)}
$$

Eq. 29 shows that loss tangent explicitly depends upon electric field since both $\widetilde{\Omega}$ and $\Gamma(\omega)$ contain electric field terms.

\section{Numerical calculations}

By using model values given in Table 1 the temperature and field dependences of shift, width, soft mode frequency and dielectric constant and loss have been calculated (Figure 1, $2 \& 3$ ) and compared with experimental results of Sandy and Jones ${ }^{9}$.

Table 1. Model values of physics parameters for Rochelle salt crystal

\begin{tabular}{cccccccccc}
\hline $\begin{array}{c}\mathrm{J}, \\
\mathrm{cm}^{-1}\end{array}$ & $\begin{array}{c}\mathrm{K}, \\
\mathrm{cm}^{-1}\end{array}$ & $\mathrm{~T}_{\mathrm{c} 1}, \mathrm{~K}$ & $\mathrm{~T}_{\mathrm{c} 2}, \mathrm{~K}$ & $\begin{array}{c}\Omega, \\
\mathrm{cm}^{-1}\end{array}$ & $\begin{array}{c}\Delta, \\
\mathrm{cm}^{-1}\end{array}$ & $\begin{array}{c}\eta, \\
\mathrm{cm}^{-1}\end{array}$ & $\begin{array}{c}\mathrm{V}_{\mathrm{ik}}, \\
\mathrm{cm}^{-1}\end{array}$ & $\begin{array}{c}\omega_{\mathrm{k}}, \\
\mathrm{cm}^{-1}\end{array}$ & $\mathrm{~A}_{\mathrm{kg}} \times 10^{17} \mathrm{erg} / \mathrm{K}$ \\
\hline 354 & 351 & 255 & 2.96 & 1.82 & 0.678 & 5.51 & 11.5 & 27.20 & 5.73 \\
\hline
\end{tabular}




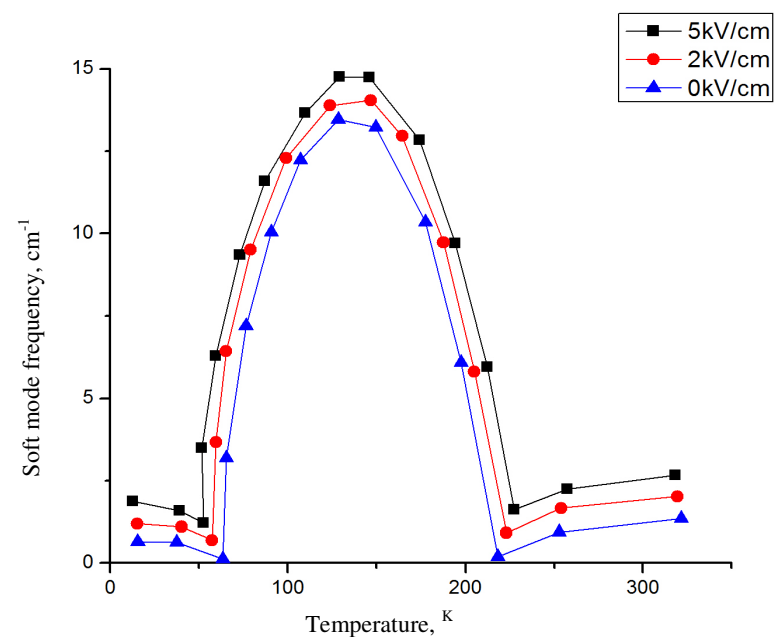

Figure 1. Calculated temperature dependence of soft mode frequency of RS with comparision of experimental data

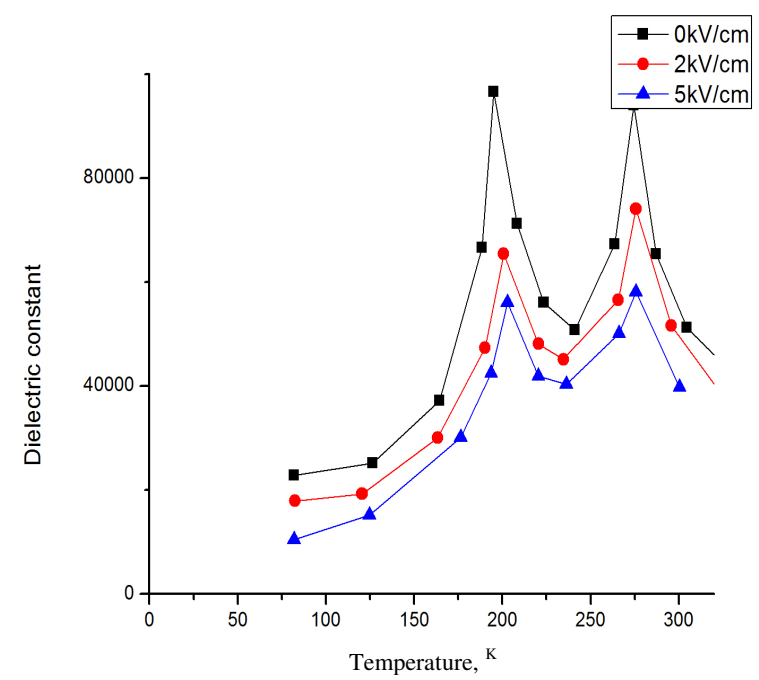

Figure 2. Calculated temperature dependence of dielectric constant of RS with comparision of experimental data

\section{Results and Discussion}

Earlier researchers ${ }^{3}$ have not considered third order phonon anharmonic interaction term. They decoupled the correlation at an early stage, due to which some important interactions disappeared from their expressions. It can be seen from our expressions that our frequency $\widetilde{\Omega}$ is the same as the initial frequency of Chaudhuri et $a l^{3}$. However, our soft mode frequency $\hat{\Omega}_{-}$contains extra terms $\Delta(\omega)$. The soft mode frequency of Chaudhuri et al., ${ }^{3}$ contains terms like $\Delta(\omega)$. But our soft mode frequency $\hat{\Omega}_{-}$contains extra term in $\widetilde{\widetilde{\omega}}_{k}$ and 
$\Gamma_{k}(\omega)$. These extra terms are $\left|V^{3}\left(k_{1}, k_{2},-k\right)\right|^{2}$ term given in $\Delta_{k}(\omega)$ in $\Gamma_{k}(\omega)$. These terms differentiate our expressions with the expressions given in the work of Chaudhuri et $a l^{3}$. The inclusion of third-order phonon anharmonic interaction terms is quite important since its inclusion gives correct experimental temperature dependence of ferroelectric and dielectric properties of Rochelle salt crystal. The phonon anharmonic interactions modify the soft mode frequency through spin-lattice interaction. The soft mode frequency increases while both dielectric constant and loss tangent decrease with the increase in the electric field strength. This finding is found to be in agreement with the experimental observations.

\section{Conclusion}

The two sublattice-pseudospin-latttice coupled mode model along with third and fourth order phonon anharmonic interaction terms explains well the temperature dependence of soft mode frequency, dielectric constant and loss tangent in Rochelle salt in the presence of electric field. Theoretical results agree well with experimental results of Sandy and Jones ${ }^{9}$.

\section{Acknoweledgement}

Authors are grateful to Eminent Physicist Prof. B S Semwal (Ex HOD Physics) for his valuable suggestions. They are also thankful to Prof U C Naithani(HOD Pauri campus), Prof R P Gairola(former HOD Srinagar campus), Prof S C Bhatt(HOD Physics), Prof K D Purohit, Prof Vinay Gupta (DelhiUniv.), Prof S K Tripathi(Punjab Univ.) and to Mrs Deepali, Mrs Anubhuti, Miss Aanchal, Mr. Prabhat Khanduri and Dr. Deepak Joshi for their valuable encouragements.

\section{References}

1. Mular K A, Helv Phys Acta, 1986, 59, 874-884.

2. Mason W P, Phys Rev., 1958, 111, 1259.

3. Chaudhuri B K, Ataki T,Ganguli S and Chihura H S, Phys Soc Jpn., 1980, 49, 609,

4. Hlinka J, Kulda J, Kamba S and Petzelt I, Phys Rev B, 2001, 63, 052102-4; DOI:10.1103/PhysRevB.63.052102

5. Shiozaki Y, Shimizu K, Nozaki R, Ferroelectrics, 2001, 261, 239-244; DOI:10.1080/00150190108216291

6. NodaN, Nozaki R and Shiszoki Y, Phys Rev B, 2000, 62, 12040; DOI:10.1103/PhysRevB.62.12040

7. Kikuta T, Kawabe R, Yamazaki T and Nakatari N, J Korean Phys Soc., 2003, 42, S1275-S1278.

8. Zubarev D N, Sov Phys Vsp., 1960, 3, 320-345; DOI:10.1070/PU1960v003n03ABEH003275

9. Sandy F and Jones R V, Phys Rev., 1968, 168, 481; DOI:10.1103/PhysRev.168.481

10. Upadhyay T C and Semwal B S, Indian J Pure Appl Phys., 2002, 40, 615.

11. Upadhyay T C, Indian J Pure Appl Phys., 2007, 45, 157. 